\title{
Fight the Fire with Mock Model Theory
}

\author{
Hongyan WANG and Zhongqian $\mathrm{SU}^{1}$ \\ Liaoning Institute of Science and Technology, China
}

\begin{abstract}
Wildfires are an important and heretofore contributor to the climate, especially in some special country,for example southeastern Australia. A large population live in this region, so the wildfires would be dangerous in the extreme. In this paper, we will study how to stop wildfires as quickly as possible. First, The fuzzy clustering analysis is used to classify forest coverage, wind velocity and population distribution. Then In the analysis model level, the elements we considered are the weight of above three factors, made up of some combination of them. In adapting to the changes that may occur in extreme fire events in the next decade, we use the Braess paradox to verify the models with the impact of climate. We supposed the scan cover of the UAV is circular function related with the flying altitude, so linear programming equation could be established at different altitude. We also use this idea to constrain some models of different terrain. It showed that the model has good adaptability with a quick convergence speed by computer simulation. The model was adopted for least-squares data fitting to process experimental data, and a high correlation coefficient was obtained.
\end{abstract}

Keywords. Cluster analysis, Hierarchical analysis, Dynamic back gauge, Braess paradox, linear programming.

\section{Introduction}

Focusing on wildfires in the southeast of Australia form October 1, 2019 to January 7,2020 , because of the fire happen in a severe drought and continuous heat weather, has a great impact on climate change adds in the around globe,Australian firefighters have uaed the medthod of drones,repeater, to monitor the site and given situational awareness (SSA), used for monitoring and reporting of the front line staff wearable equipment data, to obtain real-time environmental conditions at the foot, letting the emergency operations center EOC can balance and security and economic situation, and protect the area in a time.

Here, We only consider a rectangular area in southeastern Australia, assuming that this area meets the requirements of the question, to analyze the number of drones and the number of new departments required. When calculating the number of drones, the distance of drones is set to a maximum distance of 30 kilometers. We suppose that the relay transmits a good signal and a transmission distance is 20 kilometers.Assuming that when calculating the hover height of the drone, the data are error-free data to calculate the hover height.

\footnotetext{
${ }^{1}$ Corresponding Author, Zhongqian SU, Liaoning Institute of Science and Technology, China; E-mail: suzhongqian1732@163.com.
} 


\section{Established the Mock Model}

Under the conditions of ensuring capacity, safety and economy, the optimal number of SSA drones, radio relay drones and the location of the new department should be determined by exploring the size and frequency of fire incidents.

Based on the similitude-scaling relationships, Using cluster analysis to perform on each variable, we must clarify the similarity measure of each variable, then make mathematical models of the relationship. In the following, Through setting the variable as $\mathrm{m}_{\mathrm{j}}$, we can use the following equation to measure the similarity between the two variables[1-2]:

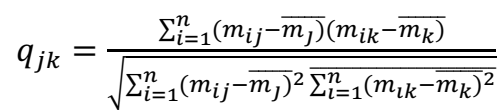

Then, we selected distance method to classify all variables by using clustering analysis.

We take the correlation coefficient matrix between the various factors as input, and take the more influential factors as the output parameters, then we can obtain the influencing factors of the three types of evaluation indicators. The first category is the impact of forest coverage, including, human influence and climate; The second category is the impact of wind speed, including air concentration and pressure; The third category is the effect of population distribution, which mainly includes fuel and oxygen concentration.

\subsection{Use Analytic Hierarchy Process to Build Mathematical Model}

We decompose this mathematical model into three levels, as shown in figure 1 . The top level is defined as the target level A, which is the key indicator that affects the size and frequency of fires; The middle level is defined as the criterion level B, which is the three main influencing factors, forest coverage area and wind speed ,Population distribution; The lowest level is designated as the program level $\mathrm{C}$, that is, seven influencing factors: terrain, human influence, climate, air concentration, air pressure, combustibles, oxygen concentration.

According to the solution of the analytic hierarchy model, we respectively get the weight of each influencing factor. It shows the proportion of each influencing factor intuitively through the bar chart. And, we can determine the rankings with the percentage of individuals factors. The most important three factors with a large proportion are selected as the branches of the index system, namely climate, air humidity and fuel.

With the data get from the internet, we get low fuel concentration, humidity and dry climate region, the distribution of the three regional division showed in figure 2 . Once we decide the state variables,such as the drone stays stationary in one position, and the drone moves over the length of the planned path, we can obtain the number of drones and the number of new units needed to be placed.

Then, we can solve the two decision variables in every progress. First of all,Supposed the maximum flight distance of the UAV is $30 \mathrm{~km}$, the information transmitted distance of the repeater is $20 \mathrm{~km}$, and we set the planned path as $622.38 \mathrm{~km}$. 


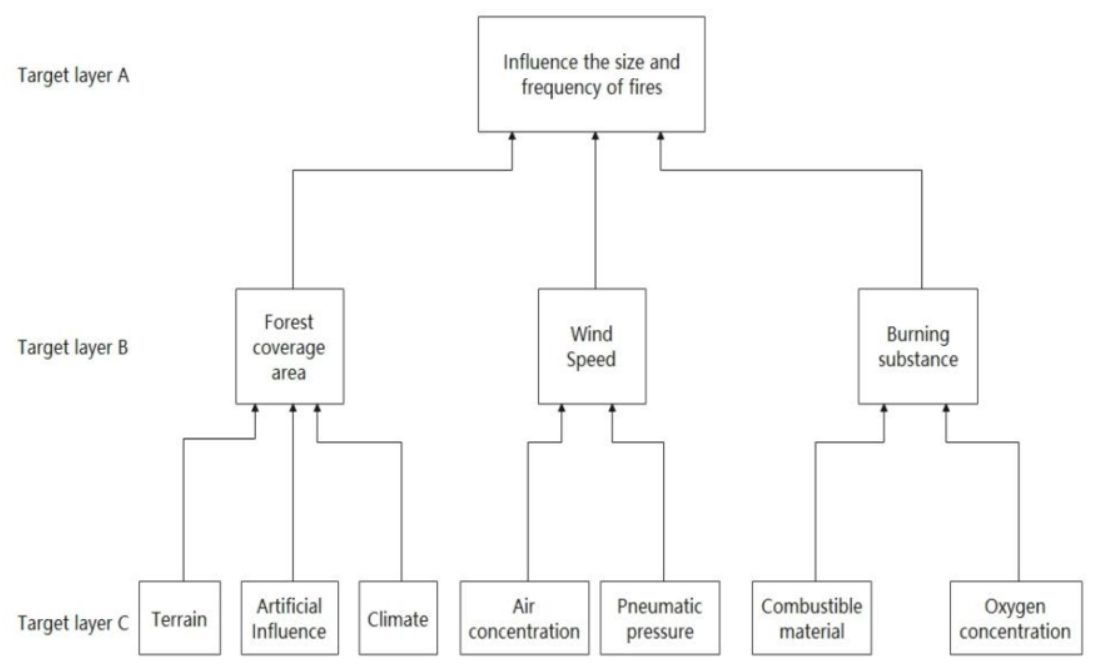

Figure 1. Analytic hierarchy diagram

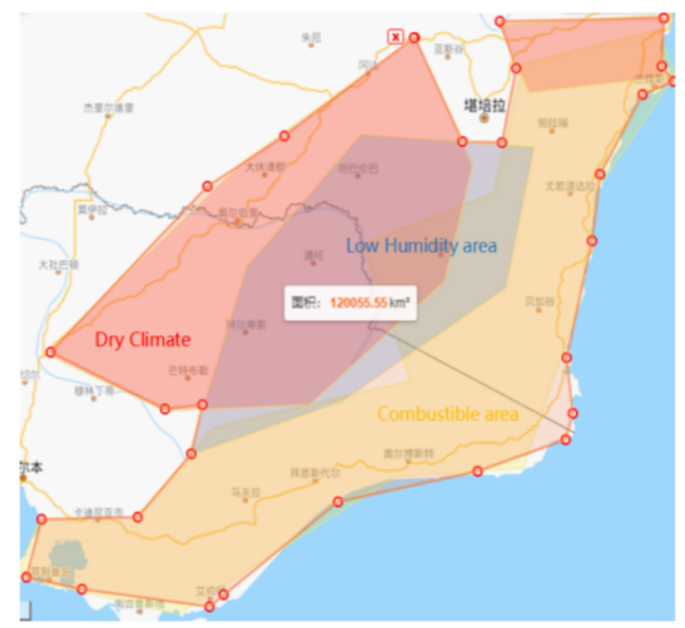

Figure 2. Climate, air humidity and fuel distribution area

By alternating the drones on even and odd number, the odd one with a repeater, the even one with a detector,and so on. Then, we fix the position of each drone.The distance between the two unmanned aerial vehicle (UAV) is $20 \mathrm{~km}$. The last not least, it is necessary to positioned the charging station at $15 \mathrm{~km}$ intervals for ensuring reciprocating flying of the UAV freely. And the distance relay transmit information of 20 kilometers. From the above convention, we set the distance between the two unmanned aerial vehicle (UAV) of 50 kilometers, each under the unmanned aerial vehicle (UAV) to establish a department (monitoring on both sides of the unmanned aerial vehicle (UAV) flight). 


\subsection{Build Two Model Theory Mathematical Model}

The Braess paradox is used to test the conjecture of extreme fire events in the next ten years[3-4]. Through the conclusion of the first question, we can conclude that the weather suddenly changes or extreme fire events occur. A drone is added to the aerial drone network to deal with extremes. We ensure economic, security and ability. In these conditions, other drones in the air will reduce the efficiency of information transmission due to sudden changes. So we have a guess on this idea: the increase in the number of drones will lead to a decrease in the rate of transmission of information by the aerial drone network, which meets the conditions for the Braess paradox.

In actual response, EOC department will send instruction to unmanned aerial vehicle (uav) to choose the shortest path for getting the front of the intelligence in a short time and transmitted to the EOC. When we consider a problem, we often only consider its subjective factors and ignore its external factors. For example: the climate is dry, which causes the humidity of the trees to decrease, the dead branches increase, and the fire becomes larger.

Passing time is:

$$
t_{\mathrm{i}}=t_{i}+n_{i} m_{i}
$$

Where,t represents the time spent in the section, $m$ represents the number of drones passing through the section, and $\mathrm{n}$ represents the delay coefficient.

When $\mathrm{n}_{3} n_{6}>=\left(n_{4}+n_{5}\right)\left(n_{1}+n_{2}\right)$, the former is less than the latter, Braess paradox will not occur.Partial transformation forms of the regression linear model were selected to sort out the quantity $\mathrm{m}_{\mathrm{i}}$ of UAV charging every 2.5 hours. Then, Matlab was used for linear fitting, and the results were as follows:

Table 1. Solving of delay coefficient

\begin{tabular}{rrrrrrr}
\hline $\mathrm{n}_{1}$ & $\mathrm{n}_{2}$ & $\mathrm{n}_{3}$ & $\mathrm{n}_{4}$ & $\mathrm{n}_{5}$ & $\mathrm{n}_{6}$ & $\mathrm{n}_{7}$ \\
\hline 0.0241 & 0.0237 & 0.0520 & 0.0138 & 0.0209 & 0.0654 & 0.0233 \\
\hline
\end{tabular}

It is calculated that $\mathrm{n}_{3} n_{6}=0.0034>=\left(n_{4}+n_{5}\right)\left(n_{1}+n_{2}\right)=0.0017$, and the UAV network model conforms to the Braess paradox.

\subsection{Linear Programming Model Establishment}

First of all, let's consider the height of the radio from the ground when the range distance of the radio is $5 \mathrm{~km}$ on the flat, unobstructed ground, and the maximum area of the ground covered by the radio UAV. we consider the area of this range as a circle.It can be intuitively seen from the above figure that when the length of the local plane is exactly the diameter of the circle, the VHF/UHF radio repeater UAV covers the largest area, and the height of the $\mathrm{VHF} / \mathrm{UHE}$ radio repeater UAV from the ground can be calculated as $1.58 \mathrm{~km}$.Next, we focus on the hovering position of the VHF/UHF radio repeater drone under the uneven terrain of a mountain or building with obstacles. 


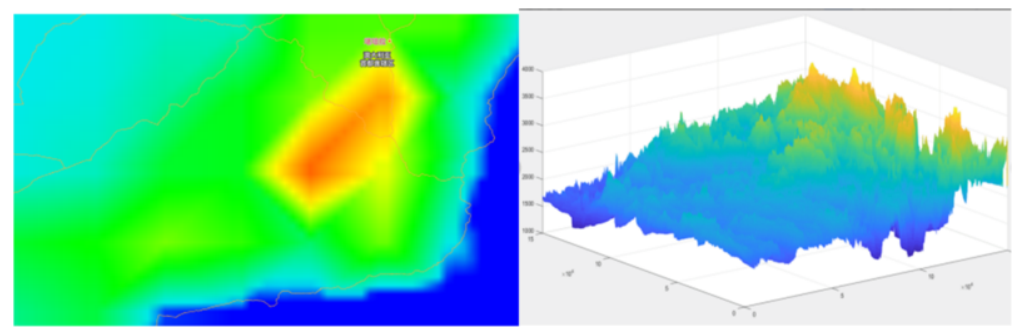

Figure 3. The distribution of terrain height

The altitude varies cause the hover position of the VHF/UHF radio repeater drone can cover the largest area of the ground. We set the range of the VHF/UHF radio repeater to $\mathrm{Lkm}$, and the height from the ground is HKm, VHF/ The UHF radio repeater UAV's range to the ground is $\mathrm{Q}$, the distance from the center of the circle to the ground is Pkm, and the distance from the center of the ground to the circle is Okm. Find out how high the VHF/UHF radio repeater is from the ground, the area covered by the ground is the largest. So, from the model build, we can get the distribution of terrain height showed in figure 3. The range of $\mathrm{VHF} / \mathrm{UHF}$ radio repeaters depends on the physical terrain of rural areas, "buildings" and "urban terrain"; weather has little effect on VHF/UHF signals. A 5 -watt radio has a the nominal range of 5 kilometers on flat, unobstructed ground, but it drops to 2 kilometers in urban areas. Australian buildings generally do not exceed 100 meters, so the range of radio will be affected in mountainous areas or high-altitude areas that are close to cities.

\section{Commentary}

From the actual situation, we consider that the drones with repeaters and hybrid drones with detectors need to charge the built-in battery multiple times under long-term work, which will cause hybrid drones damaged or unable to work, etc. Therefore, the number of UAVs with repeaters and UAVs with detectors that we choose to purchase will be more than the number of models we have established.In terms of performance, which just meet the requirements of the model.

In terms of economy, the number of new sectors can be calculated by the optimization model established by us, and the location of new sectors can be calculated by the mathematical model which has balanced the seven influencing factors of terrain, human influence, climate, air concentration, air pressure, fuel and oxygen concentration. It is the lowest cost and the best scheme in the comparison of multiple data.

\section{Acknowledgments}

The paper was supported financially by Ministry of Education, Liaoning Province.(L2019lkyfwdf-05) 


\section{References}

[1] Hanli, Mei Qiang, Lu Yumei, etc. Analysis and research of AHP-fuzzy comprehensive evaluation method [J]. China Safety Science Journal,2004, 14(7):86-89

[2] Shi Lei, Hao Tao, Yang Heng, Yang Yuan, Research on Mountain Fire Monitoring and Early Warning Algorithm of Transmission Line Based on UAV Three-light Pod [J]. Automation Technology and Application,2018,037(010):93-96

[3] HUANG Minying, MOU Rui, Improvement of Fuzzy Clustering Analysis Method and Its Application in SRM [J]. Computer Engineering and Science,2011,06:144-149

[4] ZHANG Xiu-hong, MA Ying-xue, LI Yan-hui. Analytic Hierarchy Process Based on Principal Component Analysis and Its Application [J]. Journal of Yangtze University (Natural Science Edition).2013.04:30$32+39$. 